\title{
DEPÓSITOS VOLCANICLÁSTICOS: TÉRMINOS Y CONCEPTOS PARA UNA CLASIFICACIÓN EN ESPAÑOL
}

\author{
VOLCANICLASTIC DEPOSITS: TERMINOLOGY AND CONCEPTS FOR A \\ CLASSIFICATION IN SPANISH
}

\author{
Hugo F. Murcia ${ }^{1 *}$, Carlos A. Borrero ${ }^{2}$, Natalia Pardo ${ }^{3}$, \\ Guillermo E. Alvarado ${ }^{4}$, Marcelo Arnosio ${ }^{5} \&$ Teresa Scolamacchia ${ }^{6}$
}

${ }^{1}$ The University of Auckland, School of Environment, Auckland, Nueva Zelanda ${ }^{2}$ Universidad de Caldas, Departamento de Ciencias Geológicas, Manizales, Colombia ${ }^{3}$ Massey University, Institute of Resources, Palmerston North, Nueva Zelanda ${ }^{4}$ Instituto Costarricense de Electricidad, Área de Amenazas y Auscultación Sísmica y Volcánica, San José, Costa Rica ${ }^{5}$ Universidad Nacional de Salta - Instituto Geonorte, Salta, Argentina ${ }^{6}$ Ludwig Maximilians Universität, Dept. Geo- und Umweltwissenschaften, Muenchen, Alemania *Autor para contacto: hugofmurcia@gmail.com

(Recibido: 3/10/2012; aceptado: 11/06/2013)

\begin{abstract}
The terms used to define the clastic deposits related to volcanic activity have important implications for the society in volcanic regions. Taking into account the importance of using a common terminology when considering natural hazards in the Spanish-speaking world, this study presents a classification of volcaniclastic deposits. This classification attempts to integrate the terminology related to the formational and depositional processes (eruptive and non-eruptive) taking place at volcanoes with the resulting volcaniclastic deposits and their constituent components. The classification emphasizes on how both physical and temporal variations in volcanic activity are responsible for observed differences in the related deposits. The purpose of this classification is to be a reference for the Spanishspeaking scientific community in order to avoid the numerous inconsistencies derived from the existing translations from multiple authors. Additionally, we present a flow diagram in both Spanish and English to illustrate that this classification system can be used to define and classify consolidated/lithified, and unconsolidated deposits. The appropriate classification of volcaniclastic deposits and their constituents can ultimately improve the definition and communication of potential volcanic hazards.

Keywords: volcanic terminology, volcaniclastic deposit, epiclastic deposit, juvenile clast, accessory clast, accidental clast, Latin-American examples.
\end{abstract}




\begin{abstract}
RESUMEN: La terminología empleada para definir los depósitos clásticos relacionados con la actividad volcánica, tiene importantes implicaciones para la sociedad en regiones circundantes a volcanes. Teniendo en cuenta la importancia de un lenguaje común, que se incrementa cuando se relaciona el registro geológico con las amenazas volcánicas potenciales, y la sociedad en general, en este trabajo se presenta una clasificación integral que conecta las características de los depósitos volcaniclásticos y los fragmentos constituyentes con la formación y los procesos de sedimentación (eruptivos y no eruptivos). Esta clasificación se propone como base de referencia para la comunidad científica hispanohablante con el fin de saldar las incontables inconsistencias dadas a partir de múltiples traducciones. Adicionalmente, se presenta un diagrama de flujo tanto en español como en inglés, que ilustra el sistema de clasificación integral el cual es útil para describir y definir tanto depósitos consolidados/litificados como no consolidados. Esta clasificación es útil para ser usada tanto en definición como en comunicación de la amenaza volcánica potencial.

Palabras clave: terminología volcánica, clasificación de depósitos volcaniclásticos, depósito epiclástico, fragmento juvenil, fragmento accesorio, fragmento accidental, ejemplos latinoamericanos.
\end{abstract}

\section{INTRODUCCIÓN}

En la vulcanología, como en muchas de las ramas de las ciencias geológicas, la precisión en la descripción de la estructura, textura y composición de los depósitos conlleva a la mejor comprensión de los mecanismos asociados a los eventos que intervienen en su formación, transporte y acumulación. Además, en esta rama de la geología, existe una necesidad apremiante de entender y diferenciar claramente los depósitos asociados o no a la actividad eruptiva, ya que estos no solo representan la base de los estudios geológicos y mineros sensu stricto, sino que también son fundamentales para la definición de la amenaza volcánica. Sin embargo, por ser una ciencia en pleno desarrollo con los mayores avances en los últimos decenios, en la vulcanología se ha generado una numerosa cantidad de nuevos términos, por lo general definidos inicialmente en inglés, que al ser traducidos a otras lenguas no necesariamente recogen la esencia de la definición del término original. Esta situación es reconocida para las lenguas italiana, francesa, alemana, portuguesa y castellana. En el caso de la lengua castellana, es mayor la divergencia en el uso de los significados de los términos originales, ya que por los "localismos", los términos para describir depósitos volcaniclásticos varían de país en país, tanto en Hispanoamérica como en España, ya sea en su denominación o en su significado. En este sentido, el presente trabajo es una propuesta para unificar y hacer aplicable la terminología con implicaciones genéticas, en la descripción de depósitos volcaniclásticos independientemente del país hispanoparlante, además de especificar la nomenclatura a utilizar para la denominación de tales depósitos usando los términos en lengua castellana, acorde con la evolución de la vulcanología moderna.

En este trabajo se presentan las características principales a describir en los depósitos volcaniclásticos, las características de los fragmentos que los constituyen, los tipos de clastos involucrados en los mismos según su origen de fragmentación, los tipos de depósitos según los procesos que los producen (fragmentación, transporte, acumulación) y el marco temporal que define la actividad volcánica. El trabajo presenta también unas consideraciones finales que aclaran el porqué del uso o no de algunos términos, y además se hacen otras precisiones sobre algunos términos o definiciones que en conjunto son parte fundamental de la propuesta. Para la nomenclatura y definición de los fragmentos y de los depósitos se consideraron importantes trabajos publicados sobre el tema, como son los trabajos pioneros sobre la clasificación de las rocas volcaniclásticas de Fisher (1961 y 1966) 
hasta el más reciente de Manville et al. (2009), pasando por los trabajos de Schmid (1981), Fisher \& Schmincke (1984), Cas \& Wright (1987), Fisher \& Smith (1991), McPhie et al. (1993), Martínez et al. (1996). Gillespie \& Styles (1999), Vincent (2000), Dasgupta (2003), White \& Hougthon (2006), Borrero et al. (2008), Sohn et al. (2008) y Cas et al. (2008). Adicionalmente, se hace referencia a los trabajos de Araña y Ortiz (1984), Mazzoni (1985) y Tilling (1989, traducción de Beate, 1993), los cuales introdujeron algunos términos en español actualmente muy usados en escuelas y servicios geológicos de habla hispana. Se deja a responsabilidad del lector la revisión bibliográfica detallada de la primera referencia para cada término en inglés, la cual queda por fuera del alcance de este manuscrito, para así ahondar concretamente en la unificación de la terminología. Esta propuesta incluye los depósitos volcaniclásticos hasta ahora definidos, divididos en primarios y secundarios, y separados de los depósitos epiclásticos. Es importante aclarar que en este trabajo el término general "volcaniclástico" (introducido por Fisher, 1961 y redefinido por Fisher \& Smith 1991), incluye el espectro completo de depósitos clásticos, compuestos en parte o enteramente de fragmentos volcánicos originados a partir de fragmentación mecánica por procesos volcánicos primarios o de re-sedimentación. En este contexto es importante tener en cuenta también que los términos clasto y fragmento son sinónimos.

\section{CARACTERÍSTICAS DE LOS DEPÓSITOS VOLCANICLÁSTICOS}

Los depósitos volcaniclásticos se describen siguiendo los procedimientos sedimentológicos tradicionales, considerando la identificación de capas, la geometría de las mismas, y su espesor (lámina $<1 \mathrm{~cm}$, capa muy delgada entre 1 y $3 \mathrm{~cm}$, capa delgada entre 3 y $10 \mathrm{~cm}$, capa media entre 10 y $30 \mathrm{~cm}$, capa gruesa entre 30 y $100 \mathrm{~cm}$, capa muy gruesa $>100 \mathrm{~cm}$ según Ingram (1954)), además de la continuidad y los contactos entre las mismas, es decir la estructura (tipo de estratificación si existe, gradación, estructuras sedimentarias que indiquen dirección de transporte, o cualquier característica sedimentaria relevante).

Para el caso particular de la selección de un depósito, inicialmente cuantificada por Folk \& Ward (1957), los criterios de clasificación difieren, cuando se considera la clasificación propuesta por Cas \& Wright (1987), basada en los resultados de Walker (1971), debido a que los procesos volcánicos, en los cuales el medio de transporte es gaseoso, no consiguen desarrollar el mismo nivel de selección que se logra cuando el medio de transporte involucrado es el agua. En este sentido, debería utilizarse una clasificación distinta según el medio de transporte. En el Cuadro 1 se muestra la comparación entre la clasificación de selección sedimentológica tradicional según Folk \& Ward (1957) y la referente a depósitos volcaniclásticos primarios según Cas \& Wright (1987).

Cuadro 1

Comparación entre el grado de selección de fragmentos en un depósito, considerando los criterios sedimentológicos tradicionales (Folk \& Ward, 1957) con los volcánicos propuestos por Cas \& Wright (1987)

\begin{tabular}{|c|c|c|}
\hline Selección & Folk \& Ward (1957) & $\begin{array}{c}\text { Cas \& Wright } \\
\quad(1987)\end{array}$ \\
\hline$<0,35 \Phi$ & $\begin{array}{c}\text { Muy bien } \\
\text { seleccionado }\end{array}$ & \multirow{3}{*}{$\begin{array}{c}\text { Muy bien } \\
\text { seleccionado }\end{array}$} \\
\hline 0,35 a $0,49 \Phi$ & Bien seleccionado & \\
\hline 0,50 a $0,99 \Phi$ & $\begin{array}{l}\text { Moderadamente } \\
\text { seleccionado }\end{array}$ & \\
\hline 1,00 a $1,99 \Phi$ & $\begin{array}{l}\text { Pobremente } \\
\text { seleccionado }\end{array}$ & $\begin{array}{c}\text { Bien } \\
\text { seleccionado }\end{array}$ \\
\hline 2,00 a $3,99 \Phi$ & $\begin{array}{c}\text { Muy pobremente } \\
\text { seleccionado }\end{array}$ & $\begin{array}{l}\text { Pobremente } \\
\text { seleccionado }\end{array}$ \\
\hline$>4,00 \Phi$ & $\begin{array}{l}\text { Extremadamente mal } \\
\text { seleccionado }\end{array}$ & $\begin{array}{c}\text { Mal } \\
\text { seleccionado }\end{array}$ \\
\hline
\end{tabular}




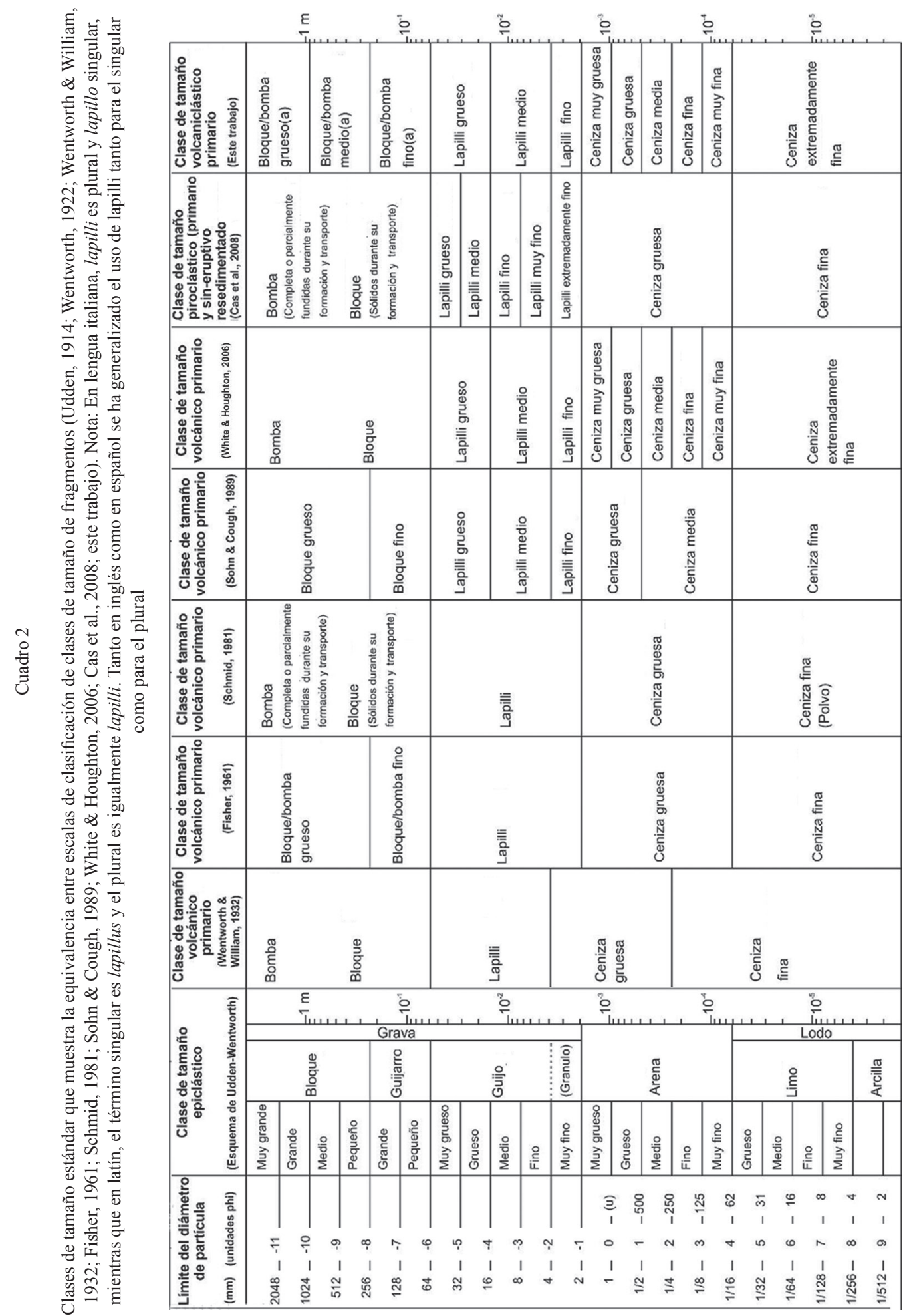




\section{CARACTERÍSTICAS TEXTURALES DE LOS FRAGMENTOS}

\section{Tamaño}

El tamaño de los fragmentos de los depósitos sedimentarios es comúnmente descrito en términos de la escala de Udden (1914), modificada por Wentworth (1922), que define los tamaños arcilla, limo, arena, gránulo, guijo, guijarro y bloque como los constituyentes de los mismos. Para depósitos volcaniclásticos se utiliza también la escala de Wentworth \& William (1932), que define los tamaños en términos de ceniza fina, ceniza gruesa, lapilli, bloques y bombas, la cual ha sido modificada por Fisher (1961), Schmid (1981), Sohn \& Cough (1989), White \& Houghton (2006), y Cas et al. (2008). En este trabajo, se introduce una nueva modificación que subdivide los fragmentos mayores a $64 \mathrm{~mm}$ (bombas/ bloques) en fino, medio y grueso, esto con el objetivo de obtener mayor consistencia en las subdivisiones independiente de los tamaños en general (ceniza, lapilli o bomba/bloque). Ambas clasificaciones, así como sus modificaciones, son óptimas para fines descriptivos, sin embargo, se recomienda utilizar la clasificación genética propuesta por Wentworth \& William (1932) modificada en este trabajo (Cuadro 2).

\section{Redondez}

La redondez de los fragmentos en un depósito se define de acuerdo con la clasificación tradicional sedimentológica propuesta por Wadell (1933) que denomina los fragmentos como muy angulares, angulares, sub-angulares, sub-redondeados, redondeados y bien redondeados. No obstante, discusiones con base en el origen o tipo no son abordadas para los fines del presente trabajo.

\section{Vesicularidad}

Un parámetro textural indispensable en la descripción de los fragmentos piroclásticos es la vesicularidad (Houghton \& Wilson, 1989; Gilbert
\& Sparks, 1998) tratada en términos de porcentaje en volumen (\%), distribución de tamaños, forma, grosor de las paredes (Navon \& Lyakhovsky, 1998), grado de conectividad (Klug \& Cashman, 1994) y modificaciones de tamaño y forma, por ejemplo por coalescencia y/o colapso (Thomas et al., 1994; Gardner et al., 1996; Klug \& Cashman, 1996). Varios de estos parámetros se obtienen en el laboratorio: los métodos más usados para la distribución de tamaños, formas y grosor de las paredes incluye el análisis de imágenes en 2D (Cashman \& Mangan, 1994; Rust \& Cashman, 2004; Shea et al., 2010) y en 3D (Giachetti et al., 2011); o mediante el uso de porosímetros de mercurio (cf., Whithman \& Sparks, 1986; Bouvet de Maisonneuve et al., 2009). El grado de interconectividad (p.e., porcentaje de vesículas conectadas y aisladas) se puede obtener con picnometría de gas (cf., Klug \& Cashman, 1994). Considerando el porcentaje de vesículas, la clasificación de Houghton \& Wilson (1989) es la más adecuada, con fragmentos no vesiculados $0 \%$, muy pobremente vesiculados $0-20 \%$, pobremente vesiculados $20-40 \%$, moderadamente vesiculados $40-60 \%$, muy vesiculados $60-80 \%$ y extremadamente vesiculados $>80 \%$. Cuando los fragmentos igualan o superan el rango de moderadamente vesiculados (40-60\%), términos como pómez o escoria son utilizados dependiendo de unas u otras características. $\mathrm{Al}$ respecto se propone hacer referencia al término pómez para denominar un fragmento de origen piroclástico compuesto por vidrio (generalmente de colores claros), vesículas y cristales (pueden o no estar presentes), poco denso (usualmente $<1 \mathrm{~g} / \mathrm{cm}^{3}$ ), en el cual la mayoría de vesículas existentes son pequeñas $(<5 \mathrm{~mm})$ y se encuentran comúnmente interconectadas y deformadas, sugiriendo magmas viscosos ricos en volátiles. Por el contrario, se propone hacer referencia al término escoria, para denominar un fragmento de origen piroclástico compuesto por vidrio (generalmente de colores oscuros), vesículas y cristales (pueden o no estar presentes), moderadamente denso (usualmente $>1 \mathrm{~g} / \mathrm{cm}^{3}$ ), en el cual la mayoría de vesículas existentes son relativamente grandes $(>5 \mathrm{~mm})$, con formas esféricas o irregulares debido a coalescencia (vesículas con paredes gruesas de donde resulta un color más 
oscuro generalmente negro, café o de tonalidad rojiza) y que de tal manera sugieren magmas poco viscosos. Esta separación se propone teniendo en cuenta las implicaciones en la explosividad del magma, donde la distribución de tamaños de vesículas (y la densidad de las mismas) está íntimamente relacionada con la intensidad de la erupción (cf., Polacci et al., 2006). Cuando la vesicularidad es superior al 98\% es apropiado usar el término reticulita (McPhie et al., 1993). Aunque los términos pómez y escoria son independientes de la composición (p.e., Mangan \& Cashman, 1996; Navon \& Lyakhovsky, 1998; Mader, 1998), generalmente la pómez está asociada a magmas ácidos e intermedios y la escoria/reticulita (cf. Rust et al., 2009) a magmas básicos. En ocasiones los fragmentos comparten características comunes o intermedias y en ese momento su definición queda a criterio del investigador.

\section{Otras características}

Una definición detallada de los fragmentos incluye la identificación y descripción de bombas y fragmentos de salpicaduras (spatter), bloques densos angulares de magma solidificado, pómez y escoria, incluyendo esquirlas de vidrio (shards) (también llamadas trizas vítreas), agregados de ceniza (cf., Scolamacchia et al., 2005; Brown et al., 2011; Van Eaton et al., 2012, y referencias citadas en estos artículos), cabellos y lágrimas de Pelé, cristales y demás fragmentos volcánicos o de origen diferente, incluyendo tanto plutónicos como metamórficos y sedimentarios.

\section{TIPOS DE FRAGMENTOS SEGÚN EL ORIGEN DE FRAGMENTACIÓN}

Múltiples definiciones y clasificaciones han sido reportadas para describir los fragmentos que componen un depósito volcaniclástico (p.e. Fisher, 1961; Schmid, 1981; Fisher \& Schmincke, 1984; Cas \& Wright, 1987; Fisher \& Smith, 1991; McPhie et al., 1993; Martínez et al., 1996; Gillespie \& Styles, 1999; Vincent, 2000; White \& Hougthon, 2006; Sohn et al., 2008; Cas et al.,
2008). Sin embargo, la discusión se ha mantenido hasta la actualidad, entre otras cosas, debido a la practicidad o no de la aplicación de los términos al momento de describir los depósitos, y a la diferencia que suscita en la aplicación dependiendo de si los depósitos son antiguos o recientes y de si involucraron agua o gas durante su transporte. Esta situación tiene un origen conceptual, en donde algunos autores definen los fragmentos volcaniclásticos acorde con el proceso que los genera y otros acorde con el proceso que los transporta (Fisher \& Smith, 1991). En este ítem, se propone una clasificación del tipo de fragmentos que componen los depósitos volcaniclásticos según su proceso de fragmentación, basados en las denominaciones de los autores mencionados anteriormente y con las apreciaciones de las experiencias prácticas de los autores del presente trabajo. Tal clasificación y sus definiciones, son aplicables para la descripción de los fragmentos, tanto en depósitos volcaniclásticos recientes como en depósitos volcaniclásticos antiguos.

En este sentido, un depósito volcaniclástico puede estar formado por uno o varios de los siguientes cinco tipos de fragmentos: piroclásticos, hialoclásticos, autoclásticos, epi-volcaniclásticos y epiclásticos. Cada uno de ellos tiene una definición en términos del origen de fragmentación mecánica como se describe a continuación:

Los fragmentos piroclásticos (pyroclastics) hacen referencia a aquellos fragmentos originados durante una erupción volcánica explosiva tanto (1) magmática (por exsolución y expansión de volátiles durante la descompresión rápida del magma), (2) hidro-magmática (por el contacto de agua externa con magma en ascenso), (3) freática o hidrotermal (por fragmentación de rocas pre-existentes a causa del calentamiento y sucesiva despresurización de agua freática o fluidos del sistema hidrotermal del volcán por parte de un cuerpo magmático). Los fragmentos piroclásticos pueden subdividirse en juveniles, accesorios y accidentales. Los fragmentos juveniles (juveniles) son aquellos formados directamente de la fragmentación de magma emitido en una erupción. Estos incluyen bombas $\mathrm{y}$ fragmentos de salpicaduras, fragmentos que indiquen magma nuevo solidificado, tales como 
bloques densos angulares, pómez y escorias, cabellos y lágrimas de Pelé. Asimismo, están incluidos todos aquellos fragmentos derivados del enfriamiento abrupto y fragmentación explosiva del magma al contacto con agua externa en erupciones hidro-magmáticas subaéreas y submarinas (p.e. Scolamacchia \& Macías, 2005 para las primeras y Schipper et al., 2010 para las segundas). Por ejemplo, esquirlas de vidrio denso o pobremente vesiculado con morfologías en bloque, en musgo y con microtexturas tales como superficies fundidas $\mathrm{y} / \mathrm{o}$ escalonadas y grietas de enfriamiento (cf. Wohletz, 1983; Heiken \& Wohletz, 1985; Büttner et al., 2002). Cristales pirogénicos, formados durante el enfriamiento $\mathrm{y}$ ascenso del magma en erupción, también entran dentro de esta categoría. Los fragmentos accesorios (accesories) son aquellos volcánicos comagmáticos pre-existentes, involucrados desde el conducto, el edificio volcánico o los depósitos asociados a éste, durante una erupción (p.e. incorporados de las paredes de conducto o fuera de él durante el transporte). Un fragmento juvenil y uno accesorio pueden ser muy similares $y$, por ende, puede no ser posible su diferenciación. Por ejemplo, en un depósito, una bomba es juvenil siempre y cuando se haya formado en la erupción que dio origen al depósito, sin embargo, si la bomba es reciclada por otra erupción o proceso sedimentario, deja de ser juvenil aunque su apariencia lo sea, y se convierte en accesorio. En este caso no es posible su diferenciación, a menos que el depósito en el cual el fragmento accesorio se encuentre sea muy diferente al original y que el investigador logre diferenciarlo por composición o aspecto físico del fragmento. Los fragmentos accidentales (accidentals) son aquellos provenientes de rocas metamórficas, rocas o depósitos sedimentarios, o rocas o depósitos ígneos no co-magmáticos e involucrados durante una erupción.

Los fragmentos hialoclásticos (hialoclastics) hacen referencia a fragmentos formados por enfriamiento rápido y consecuente fragmentación pasiva de flujos de lava en movimiento o domos de lava en crecimiento al contacto inmediato con el agua o hielo (generalmente en erupciones subacuáticas o subglaciares, aunque también en productos subaéreos que entran en contacto con cuerpos de agua).

Los fragmentos autoclásticos (autoclastics) hacen referencia a fragmentos formados por la fragmentación de flujos de lava en movimiento o domos de lava en crecimiento al contacto inmediato con el aire.

Los fragmentos epi-volcaniclásticos (epivolcaniclastics) hacen referencia a fragmentos de composición volcánica, fragmentados exclusivamente debido a procesos de alteración meteórica física (erosión) o química, los cuales son transportados por agua, hielo, viento o gravedad. Cuando tales fragmentos tienen una composición diferente a la volcánica, se denominan simplemente epiclásticos (epiclastics).

En algunos depósitos, es posible que no se puedan diferenciar los fragmentos piroclástico, hialoclástico y autoclástico de un fragmento epivolcaniclástico.

En las figuras 1A (en español) y 1B (en inglés) se presenta un diagrama de flujo que ilustra el origen de cada una de los fragmentos.

\section{TIPOS DE DEPÓSITOS SEGÚN SU ORIGEN}

Similar a lo que sucede en la descripción de los fragmentos que componen los depósitos volcaniclásticos, la definición de éstos últimos ha suscitado múltiples denominaciones dependiendo de su origen con respecto al proceso de fragmentación que los produjo (p.e. si este está o no asociado a una erupción, y en caso de que lo esté, del tiempo transcurrido entre la erupción y el proceso de origen/sedimentación -ver Fisher, 1961; Schmid, 1981; Fisher \& Schmincke, 1984; Cas \& Wright, 1987; McPhie et al., 1993; Martínez et al., 1996; Gillespie \& Styles, 1999; Vincent, 2000; Dasgupta, 2003; White \& Hougthon, 2006; Sohn et al., 2008; Borrero et al., 2008; Manville et al., 2009). En este ítem se propone una clasificación para denominar los tipos de depósitos volcaniclásticos teniendo en cuenta su origen y que además pueda aplicarse tanto a depósitos recientes como a depósitos antiguos. Tal clasificación 


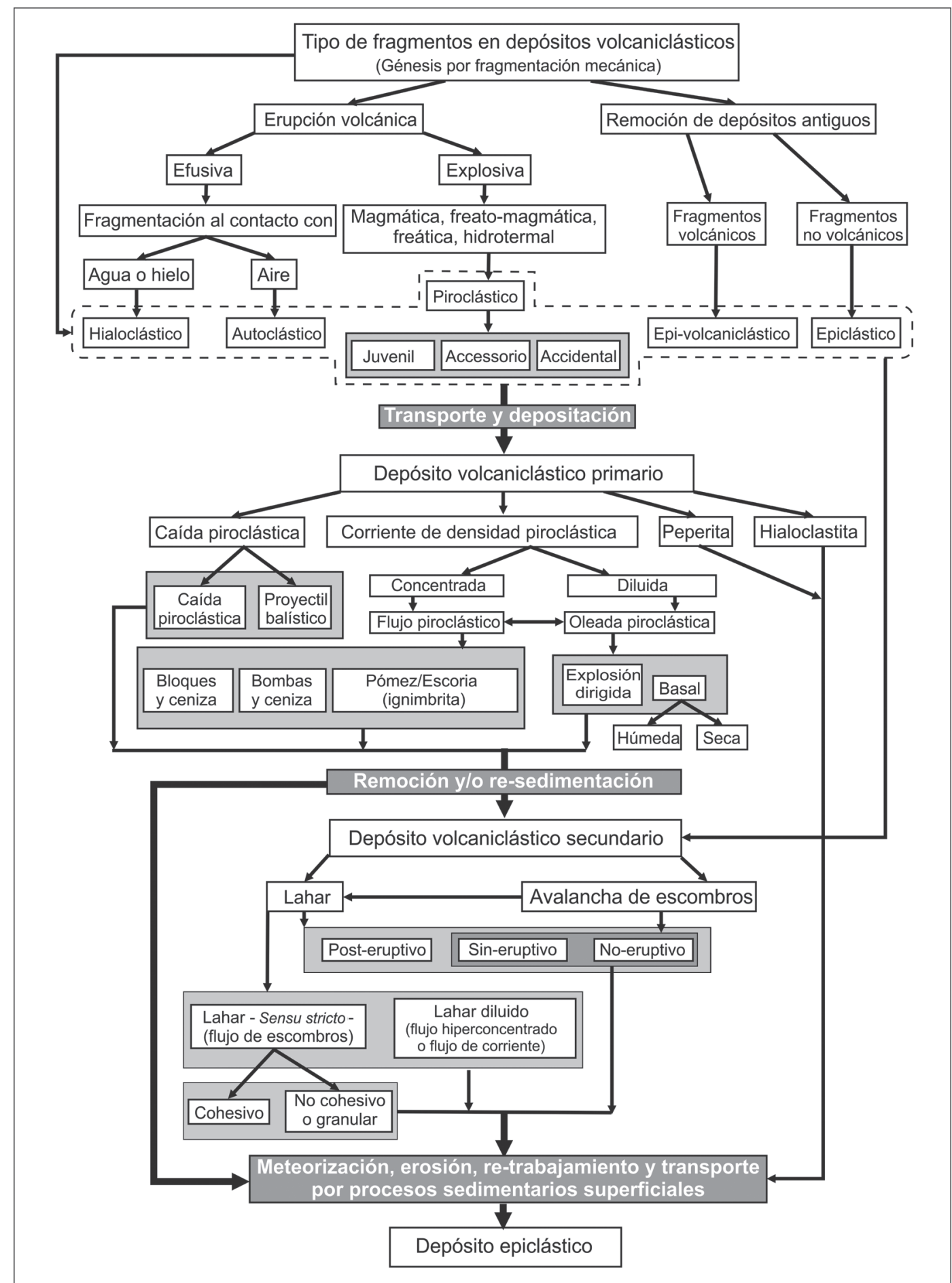

Fig. 1A: Diagrama de clasificación que ilustra los tipos de fragmentos que forman un depósito volcaniclástico, y los tipos de depósitos volcaniclásticos primarios y secundarios. 


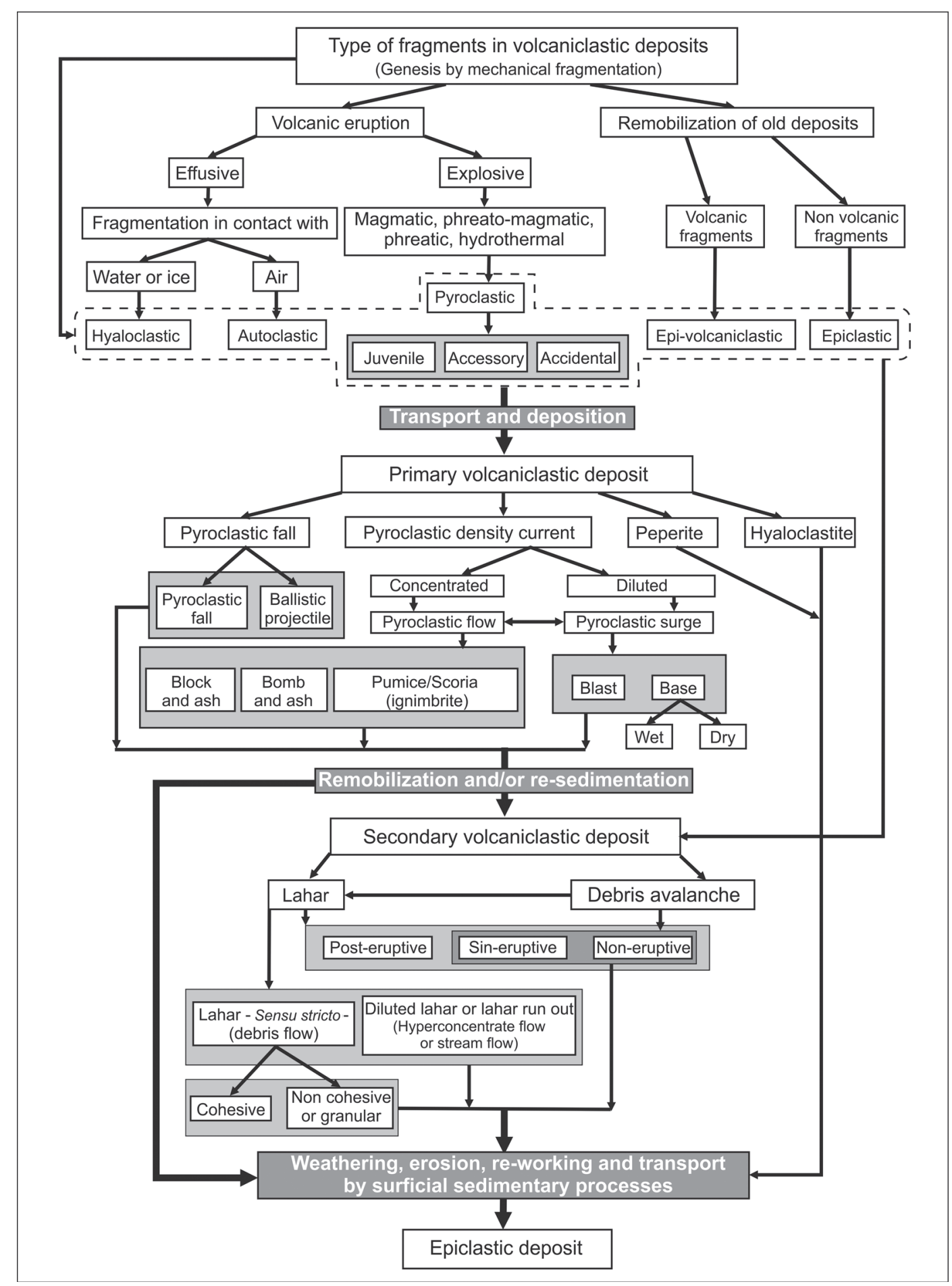

Fig. 1B: Traducción al inglés de la Fig. 1A: Flow diagram illustrating the types of fragments that form a volcaniclastic deposit, and the types of primary and secondary volcaniclastic deposits. 
incluye los depósitos volcaniclásticos (diferenciados de los depósitos epiclásticos) como se describe a continuación:

Un depósito se denomina volcaniclástico primario (primary volcaniclastic) si sus facies indican una acumulación que ocurre a partir de una caída piroclástica, de una corriente de densidad piroclástica $o$ de una peperita, y si los fragmentos predominantes son piroclásticos (juveniles, accesorios o accidentales) o autoclásticos. En el caso particular de depósitos clásticos formado por fragmentos hialoclásticos, el término hialoclastita es necesario para diferenciar su origen. Un depósito se denomina volcaniclástico secundario (secondary volcaniclastic) si sus facies indican una acción gravitacional, o agentes como el agua, viento y/o el hielo actuando directamente en procesos de remoción y sucesiva re-sedimentación (avalanchas de escombros volcánicas y lahares) a partir de un depósito volcánico; en este caso los fragmentos pueden ser de cualquier tipo (piroclásticos -juveniles, accesorios o accidentales-, hialoclásticos, autoclásticos, epi-volcaniclásticos y epiclásticos). Un depósito se denomina epiclástico (epiclastic) si las facies indican remoción por procesos meteóricos (no asociadas a la actividad volcánica) de cualquier tipo de roca (p.e. ígnea, metamórfica o sedimentaria) o depósito pre-existente y además los fragmentos predominantes son epiclásticos o epi-volcaniclásticos. En algunos depósitos es posible que no se pueda definir si se trata de un depósito volcaniclástico secundario o de un depósito epiclástico con fragmentos epi-volcaniclásticos. En este caso se recomienda la búsqueda de otros criterios que indiquen la relación directa con el vulcanismo.

\section{Definiciones}

Una acertada definición de los depósitos volcaniclásticos, es la base indispensable para entender los mismos en términos de sus implicaciones genéticas. En este sentido, la observación de los procesos volcánicos y la evolución de la vulcanología como disciplina han permitido diferenciar tipos de depósitos volcaniclásticos con base en su origen, además de sus implicaciones directas con respecto al medio de transporte y a los fragmentos involucrados. Acorde con las definiciones tratadas en los ítems anteriores, en este apartado se hace énfasis en los conceptos que enmarcan cada tipo de depósito volcaniclástico y a su vez en sus constituyentes, como se describe a continuación (Figs. 1A y 1B).

Un depósito de caída piroclástica (pyroclastic fall) es el producido por una lluvia de fragmentos piroclásticos (juveniles, accesorios o accidentales) que por definición tienen su origen en una erupción volcánica (Figs. 2A y 2B). Los fragmentos se acumulan por gravedad desde la nube o pluma de cenizas desarrollada al tope de una columna eruptiva. Cuando los fragmentos son lanzados siguiendo una trayectoria parabólica desde el volcán, sin ser incorporados en la zona convectiva de la columna eruptiva, se denominan proyectiles balísticos (ballistic projectiles) (Figs. 2C y 2D).

Un depósito de corriente de densidad piroclástica (pyroclastic density current) hace referencia al depósito producido por un flujo de fragmentos, gases y vapor en fase de condensación u agua líquida (flujo con una densidad mayor que la del aire) que se mueve sobre un terreno bajo la influencia de la gravedad y que tiene su origen en una erupción volcánica (ver Fisher, 1966; Druitt, 1998; Branney \& Kokelaar, 2003). Los fragmentos constituyentes son piroclásticos (juveniles, accesorios o accidentales) o autoclásticos con o sin presencia de fragmentos epiclásticos o epivolcaniclásticos. Los depósitos de corrientes de densidad piroclástica se generan a partir de flujos con alta concentración de fragmentos (corriente de densidad piroclástica concentrada -concentrated pyroclastic density current-) ( $\geq 10 \%$ en volumen, Freundt \& Bursik, 1998) o de flujos con baja concentración de fragmentos (corriente de densidad piroclástica diluida -diluted pyroclastic density current-) (1-10\% en volumen) (Wilson \& Houghton, 2000; Valentine \& Fisher, 2000; Branney \& Kokelaar, 2003). De esta manera los flujos concentrados forman depósitos también llamados de flujo piroclástico (pyroclastic flow) mientras que los flujos no concentrados o diluidos forman depósitos también llamados de oleada piroclástica (pyroclastic surge). Cuando los depósitos presentan características de ambos tipos se recomienda conservar el término corrientes de densidad piroclástica (Figs. 3 y 4). 



Fig. 2: Depósito de caída piroclástica. A) Caída piroclástica de pómez (volcán Doña Juana, Colombia). B) Caída piroclástica de escorias de la erupción de 1723 (volcán Irazú, Costa Rica). C y D). Proyectiles balísticos (C, bomba fusiforme, volcán Tecuitlapa, México; D, bloque juvenil, volcán Irazú, Costa Rica).

Es importante tener en cuenta que los depósitos de corrientes de densidad concentradas (p.e., los flujos piroclásticos), pueden presentar facies asociadas con partes diluidas del flujo como tal, sin embargo, tales facies no hacen referencia a depósitos de oleadas piroclásticas por sí mismas.

A su vez, los depósitos de flujo piroclástico se denominan depósitos de flujo de bloques y ceniza (block and ash flow) si están asociados al colapso gravitacional y/o explosivo de domos o flujos de lava, o al colapso de una columna eruptiva que involucra la destrucción de los mismos (ver Freundt et al., 2000), y está compuesto principalmente de fragmentos piroclásticos (juveniles, accesorios o accidentales) o autoclásticos (no se excluye la posibilidad de que contenga pómez o escoria) (Fig. 3A); depósitos de flujo de bombas y ceniza (bomb and ash flow) si el proceso de formación involucra lava fundida emitida en forma de bombas (ver
Alvarado \& Soto, 2002; Miyabuchi et al., 2006) (Fig. 3B); o depósito de flujo de pómez (pumice flow) o ignimbrita (ignimbrite) si está asociado al colapso gravitacional de una columna eruptiva generada a cráter abierto o a procesos de desbordamiento por ebullición (boiling over) (Smith, 1960; Spark et al., 1973; Fisher \& Schmincke, 1984; Cas \& Wright, 1987; Freundt et al., 2000; Branney \& Kokelaar, 2003) y por ende, está compuesto principalmente de fragmentos de pómez (independientemente del tamaño de los fragmentos y del grado de soldadura del depósito) (Fig. 3C, 3D y 3E). Si los principales fragmentos constituyentes del depósito de flujo piroclástico son definidos como escoria, entonces el depósito se denomina de flujo de escoria (scoria flow) (Druitt, 1998) (Fig. 3F).

Los depósitos de oleadas piroclásticas se denominan depósitos de oleadas basales (base surges) si son producidos por la depositación 

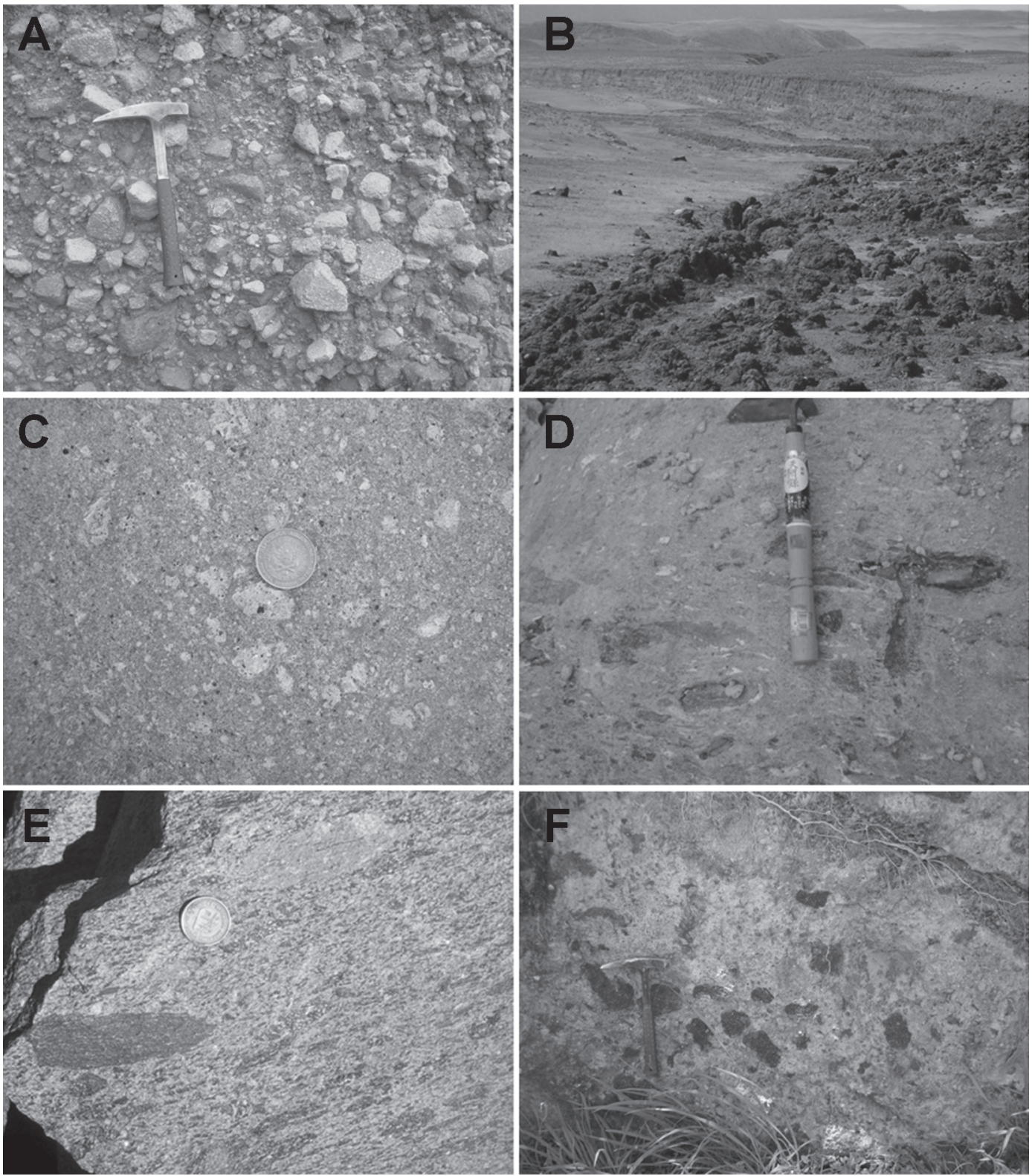

Fig. 3: Depósitos de corriente de densidad piroclástica concentrada. A) Facies de flujo piroclástico de bloques y ceniza (volcán Azufral, Colombia). B) Facies de flujo piroclástico de bombas (volcán Cotopaxi, Ecuador). C) Facies de flujo piroclástico de pómez o ignimbrita no soldada (volcán Tuzgle, Argentina). D y E) Facies de fujos piroclásticos de pómez con diferente grado de soldadura (D, Guanacaste, Costa Rica y E. Ignimbrita Vizcachayoc, Argentina). F) Facies de flujo piroclástico de escoria (sector norte del abanico de Lérida, Tolima, Colombia).

de corrientes de densidad generadas desde una columna eruptiva durante erupciones freatomagmáticas o hidro-magmáticas y freáticas (Fig. 4A). Por lo general, este tipo de oleadas se desarrollan desde la base y por colapso (cf. Wohletz, 1998) de columnas eruptivas bajas y propulsiones a chorro (jets), por lo que la energía cinética y alcance son menores de aquellas 



Fig. 4: Depósitos de corriente de densidad piroclástica diluida. A) Facies de oleadas piroclásticas basales (volcán Sotará, Colombia). B) Facies de oleada piroclástica de explosión dirigida (volcán Doña Juana, Colombia).

asociadas a flujos piroclásticos. Generalmente, estos depósitos forman estructuras de conos y anillos de toba y maares (Moore, 1967; Fisher \& Waters, 1970; Crowe \& Fisher, 1973), pero también se generan por fases freáticas y freatomagmáticas de cualquier tipo de volcán (p.e., Nairn et al., 1979; Scolamacchia \& Macías, 2005). Tales oleadas se denominan húmedas (wet surges) si son depositadas a una temperatura menor a $100^{\circ} \mathrm{C}$, y por tanto involucran agua en estado líquido (o vapor en fase de condensación), o secas (dry surges) si son depositadas a una temperatura mayor a $100^{\circ} \mathrm{C}$ (Sheridan \& Wohletz, 1983), sin presencia de agua líquida. Cabe notar que la condensación del agua puede generar una variación longitudinal de oleadas secas a oleadas húmedas con la distancia desde la fuente. Los depósitos de oleadas asociadas a explosiones laterales debido a la despresurización de un criptodomo (Kieffer, 1981; Fisher et al., 1987; Belousov, 1996; Alvarado et al., 2006) se denominan depósitos de oleadas de explosión dirigida (blast surges) (Fig. 4B).

Un depósito peperítico o simplemente una peperita (peperite) es el/la producido/a por el contacto de magma/lava (o de corrientes de densidad piroclástica con comportamiento similar) con sedimentos no consolidados tanto secos como húmedos o saturados en agua (Fisher, 1961; White et al., 2000; Skilling et al., 2002; Jerram \& Stollhofen., 2002; White \& Houghton, 2006; Petry et al., 2007; Waiche et al., 2007; Branney et al., 2008; Németh \& White, 2009), y en los fragmentos constituyentes pueden encontrarse fragmentos piroclásticos, hialoclásticos, epiclásticos y/o epi-volcaniclásticos (Fig. 5).

Un depósito hialoclastítico o hialoclastita (hyaloclastite) es el/la producido/a por una extensa fragmentación pasiva al contacto magma/lava con grandes cuerpos de agua o hielo (ver Watton et al., 2012) y los fragmentos constituyentes son principalmente hialoclásticos (Fig. 6).

Un depósito de avalancha de escombros volcánica (volcanic debris avalanche) es un depósito producido por el flujo debido a un colapso de una parte del edificio volcánico, el cual está o no asociado a una erupción (Siebert, 1984; Ui et al., 2000). Los fragmentos constituyentes pueden ser de cualquier tipo (piroclásticos, hialoclásticos, autoclásticos, epi-volcaniclásticos y epiclásticos). (Fig. 7A y 7B). Si la avalancha está asociada a una erupción, el depósito se denomina de avalancha volcánica sin-eruptiva (syn-eruptive debris avalanche), y si está asociado a un sismo o a un mecanismo disparador desconocido, el depósito se denomina de avalancha volcánica no-eruptiva (non-eruptive debris avalanche).

Un depósito de lahar (lahar) hace referencia al producido por un flujo denso de fragmentos y agua, que desciende desde un volcán debido a la remoción de los fragmentos presentes en depósitos volcaniclásticos (Smith \& Fritz, 1989; Rodolfo \& Arguden, 1991; Smith \& Lowe, 1991; 

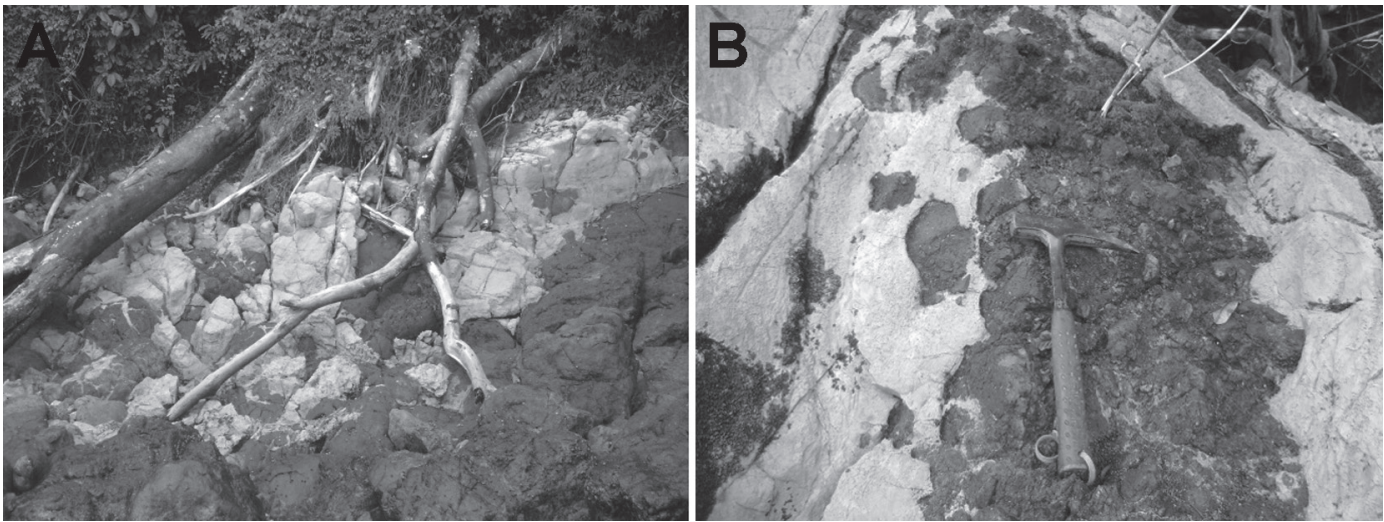

Fig. 5: Peperita (Gofito, Costa Rica). A) Afloramiento. B) Detalle del depósito.

Vallance, 2000). Los fragmentos constituyentes pueden ser de cualquier tipo (piroclásticos, hialoclásticos, autoclásticos, epi-volcaniclásticos y epiclásticos). Si el lahar es inducido por una erupción, el depósito se denomina depósito de lahar sin-eruptivo (syn-eruptive lahar), pero si el lahar es inducido por factores externos posteriores a la erupción, el depósito se denomina depósito de lahar post-eruptivo (post-eruptive lahar), y si el lahar no está asociado a una erupción se denomina depósito de lahar no-eruptivo (noneruptive lahar). Para depósitos de lahar antiguos puede ser difícil, y a veces imposible, diferenciar su origen específico, por lo que se sugiere conservar simplemente el término depósito de lahar. Independiente de esta subdivisión, si la concentración de fragmentos es alta (factor directamente relacionado con su comportamiento reológico), el flujo originado se denomina lahar sensu stricto (Fig. 8A) o flujo de escombros volcánico (volcanic debris flow), de lo contrario se denomina lahar diluido (diluted lahar or lahar run out), que puede comportarse reológicamente como un flujo hiperconcentrado volcánico (volcanic hyperconcentrated flow) (ver Pierson, 2005 para límites superiores e inferiores de concentración de sedimentos y otros factores importantes) o como un flujo de corriente (stream flow


Fig. 6: Depósitos de lavas en almohadilla e hialoclastitas. A) Lavas almohadilladas con hialoclastitas inter-almohadas (Punta Burica, Costa Rica). B) Depósito de hialoclastitas (Quepos, Costa Rica). 

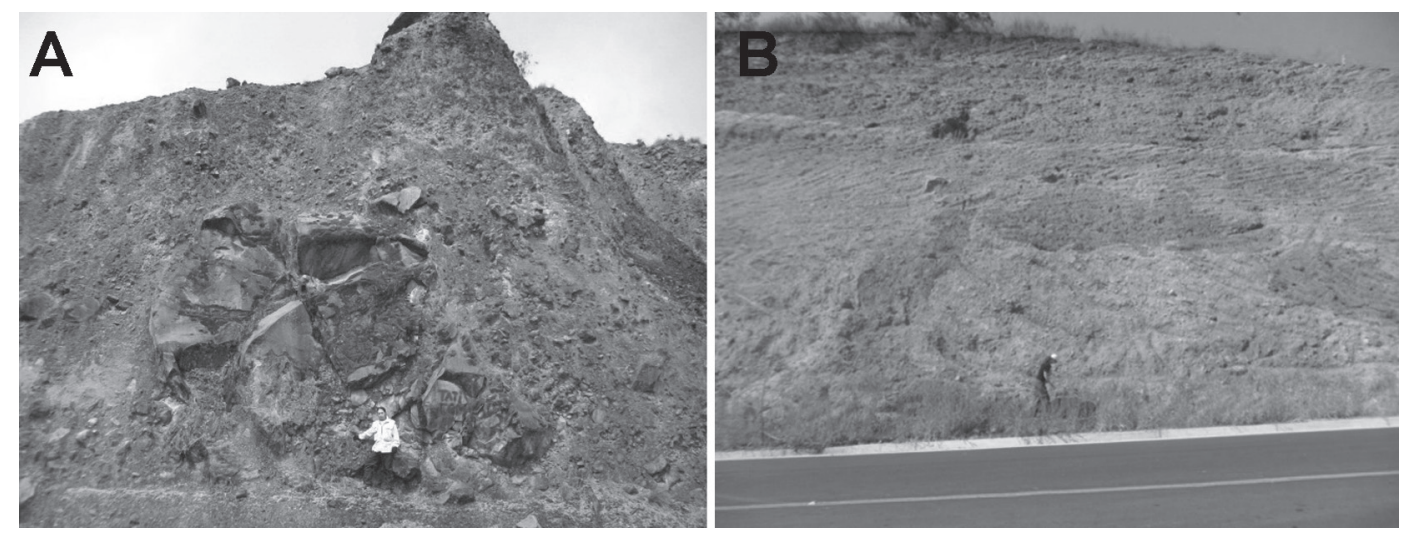

Fig. 7: Depósitos de avalancha de escombros volcánica. A) Mesobloque con estructura en rompecabezas (volcán Nevado del Ruiz, Colombia). B) Bloques deformados (volcán Colima, México).

or normal streamflow) (Iverson, 1997; Vallance \& Scott, 1997; Iverson \& Vallance, 2001). (Fig. $8 \mathrm{~B})$. Finalmente, si un depósito de lahar tiene más del $5 \%$ en peso de arcilla en la matriz se denomina cohesivo (cohesive) y si tiene menos se denomina no cohesivo (non-cohesive) o granular (granular) (Vallance \& Scott, 1997).

Como se mencionó anteriormente, un depósito se denomina volcaniclástico secundario (secondary volcaniclastic) si sus facies indican una acción gravitacional, o agentes de transporte como el agua, viento y/o el hielo actuando directamente en procesos de remoción y sucesiva re-sedimentación (avalanchas de escombros volcánicas y lahares) a partir de un depósito volcánico; en este caso los fragmentos pueden ser de cualquier tipo (piroclásticos -juveniles, accesorios o accidentales-, hialoclásticos, autoclásticos, epi-volcaniclásticos y epiclásticos) y representar cualquier porcentaje dentro del depósito. La figura 9, por ejemplo, muestra fragmentos epi-volcaniclásticos dentro de una secuencia fluvio-lacustre (Fig. 9A) y dentro de una fluvial (Fig. 9B).

En las figuras 1A (en español) y 1B (en inglés) se presenta un diagrama de flujo que ilustra la clasificación de cada uno de los depósitos.
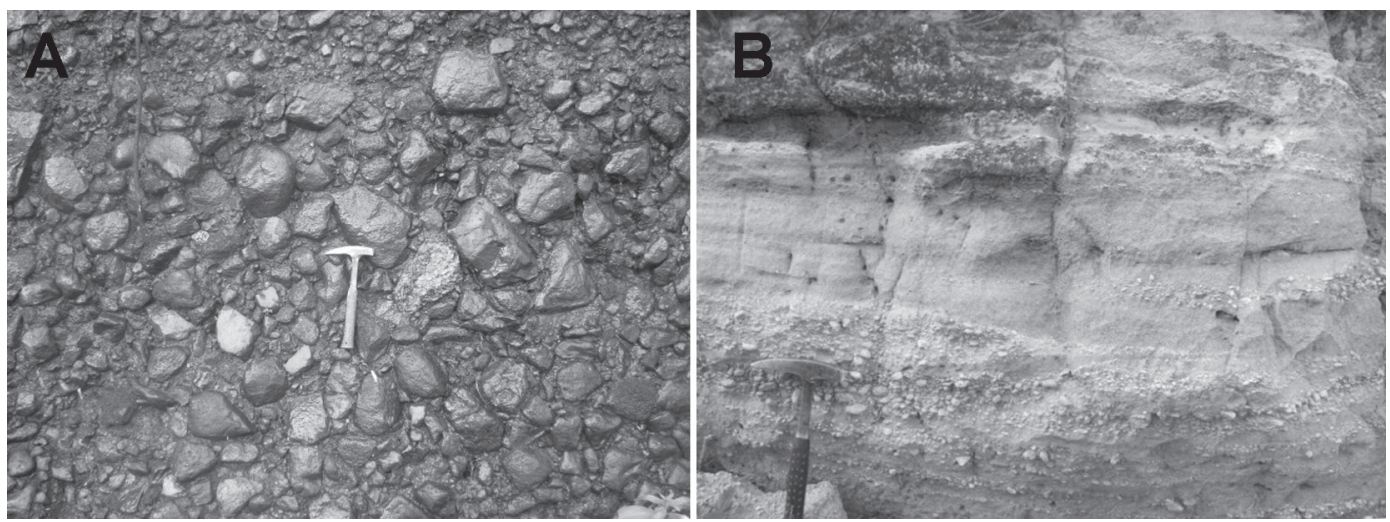

Fig. 8: Depósito de lahar. A) Facies de lahar sensu stricto (volcán Tacaná, México). B) Facies de lahar diluido (volcán Sotará, Colombia). 

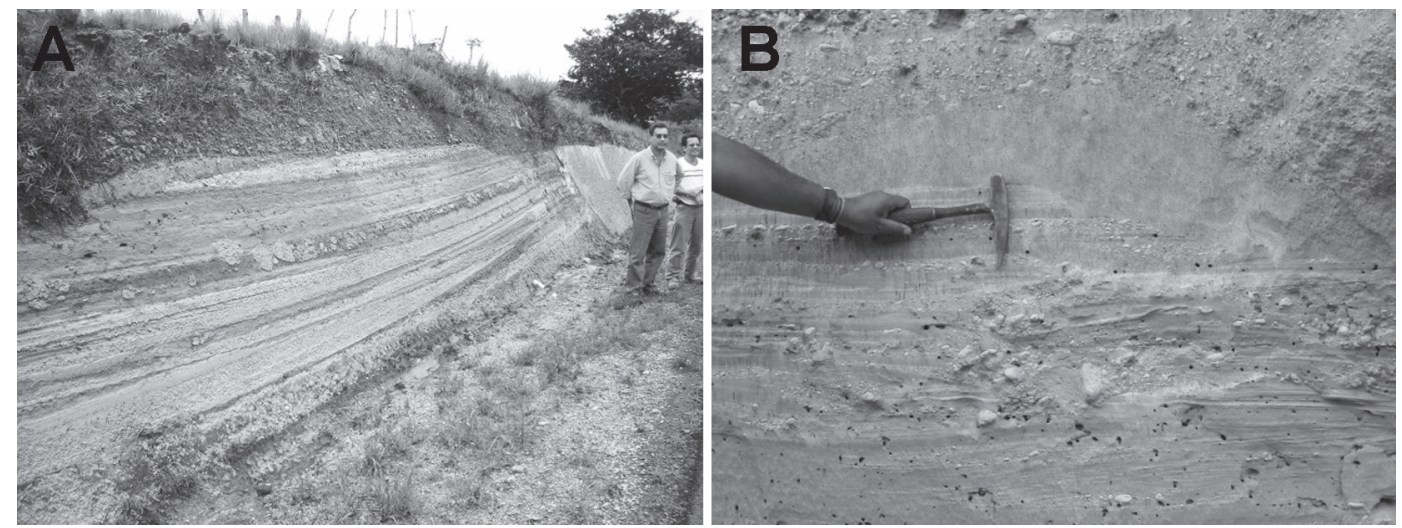

Fig. 9: Depósitos epi-volcaniclásticos. A) Facies fluvio-lacustres (La Ese, volcán Miravalles, Costa Rica).B) Facies fluviales (Tolima, Colombia).

\section{MARCO TEMPORAL DE LA ACTIVIDAD VOLCÁNICA}

Los depósitos volcaniclásticos conforman una unidad eruptiva si son acumulados entre dos paleosuelos o superficies erosivas, las cuales representan un tiempo importante de erosión y/o no acumulación. En términos de actividad volcánica, una unidad eruptiva como unidad física es acumulada durante una erupción y está formada por uno o varios depósitos relacionados a fases y pulsos eruptivos; entre ellos se pueden identificar diastemas que corresponden a periodos muy cortos de erosión y/o no acumulación. Una erupción dura minutos, horas, días o años y puede contener varias fases eruptivas que duran entre minutos y días, y que a su vez involucran varios pulsos eruptivos que duran entre segundos y minutos. Varias erupciones constituyen un periodo eruptivo que dura entre decenas, cientos y miles de años, y a su vez varios periodos conforman una época eruptiva que dura entre miles y millones de años (Fisher \& Schmincke, 1984; Freundt \& Schmincke, 1985; Schmincke \& van den Bogaard, 1991).

\section{CONSIDERACIONES FINALES}

Amplias discusiones han sido abordadas en torno a denominar tanto los depósitos volcaniclásticos como los fragmentos que los conforman. Tales discusiones son apenas obvias teniendo en cuenta que una definición y/o clasificación tiene implicaciones en términos de orígenes y mecanismos de sedimentación, tanto de los fragmentos como de los eventos volcánicos generadores de los depósitos volcaniclásticos y de las características físicas que los enmarcan. En este sentido, en diferentes trabajos es común que usen diversos términos que pueden estar indicando interpretaciones similares o viceversa. En este trabajo se abordaron los términos y conceptos con implicaciones de facies para la descripción y clasificación textural, composicional y de origen de los depósitos volcaniclásticos, con el objetivo de proponer un vocabulario práctico y cercano a la realidad, para unificar los términos en lengua castellana y a su vez, que esté acorde con los avances de los estudios vulcanológicos a nivel mundial. Para tal propuesta, se definieron y enmarcaron los depósitos y los distintos tipos de fragmentos con base en el mecanismo de fragmentación, y se ampliaron los conceptos o rangos de definiciones dentro de un marco genético debido a su implicación en la interpretación de la dinámica de las erupciones (mecanismos eruptivos, y transporte y acumulación de los productos volcánicos) y en el establecimiento adecuado de los eventos que definen las amenazas volcánicas.

Términos como depósito de ceniza (ash deposit), depósito de lapilli (lapilli deposit), y sus equivalentes litificados de toba (tuff), lapillita (lapillistone), toba lapillítica (lapilli-tuff), brecha volcánica (volcanic breccia), brecha piroclástica 
(pyroclastic breccia), brecha tobácea (tuff-breccia) y aglomerado (agglomerate), entre otros, son también utilizados para denominar depósitos volcaniclásticos (Fisher, 1961, 1966; Pettijohn, 1973). Estos términos son texturales, granulométricos y petrográficos, pero no son genéticos como los acá utilizados.

Fragmento hidroclástico (hydroclastic) es un término comúnmente usado para diferenciar un fragmento piroclástico generado cuando una erupción explosiva es producida por el contacto agua/magma. Este término mencionado por Fisher \& Smith (1991), aunque está asociado a un mecanismo de fragmentación diferente, es producido directamente por una erupción explosiva y en ocasiones no es posible de separar de uno generado exclusivamente por fragmentación magmática (p.e., una erupción pliniana -freatopliniana?, ultra-pliniana?-). En este sentido se recomienda evitar el término fragmento hidroclástico y denominarlo fragmento piroclástico juvenil haciendo énfasis en que el mecanismo de fragmentación de magma fue debido al contacto explosivo con el agua (p.e., hidro-magmático). Para los fragmentos producidos en erupciones efusivas al contacto con el agua existe el termino hialoclástico (como fue definido anteriormente). Estos mismos criterios son utilizados para recomendar el no uso del término corrientes de densidad hidroclástica (hydroclastic density current) mencionado en algunos trabajos.

El término cognato (cognate) ha sido utilizado normalmente para definir los fragmentos comagmáticos. No obstante, su definición es diferente en textos ampliamente conocidos como los de Pettijohn (1973), Fisher \& Schmincke (1984) y Cas \& Wright (1987), ocasionando un amplia confusión. Para Pettijohn (1973), los fragmentos cognatos son tanto los fragmentos esenciales (op. cit) como los accesorios siendo los primeros definidos como los generados desde el magma ascendente (p.e. juveniles) y los segundos como los generados desde erupciones previas (p.e. comagmáticos). Para Fisher \& Schmincke (1984) cognatos hace referencia exclusivamente a los fragmentos co-magmáticos y el término es sinónimo de accesorio (no se incluyen los esenciales de Pettijohn). Para Cas \& Wright (1987) cognato es un fragmento lítico magmático juvenil no vesiculado, diferente a uno accesorio que hace referencia a un fragmento involucrado de la roca caja (p.e. accidental de Pettijohn y también de Fisher \& Schmincke). En el presente trabajo, el término no es considerado debido a que los demás definidos aquí (p.e. piroclásticos -juveniles, accesorios, accidentales-, hialoclásticos, autoclásticos, epivolcaniclásticos y epiclásticos) agrupan todo el espectro de los fragmentos en términos de su origen y de su posibilidad de diferenciación tanto en los depósitos recientes como antiguos. El término cognato entonces, no es necesario, y el no usarlo evitaría más confusiones.

En la actualidad, corrientes de densidad piroclástica concentrada y diluida, son términos que claramente subdividen las corrientes de densidad teniendo en cuenta la concentración de fragmentos en el flujo. Sin embargo, una nueva subdivisión (Doronzo, 2012) sugiere abandonar estos términos y usar corrientes de densidad dominadas por fuerzas de convección (forced convection-dominated) y dominadas por inercia (inertia-dominated), teniendo en cuenta evidencias de las características del flujo. Esta situación es un claro ejemplo del dinamismo que aún existe en la terminología vulcanológica debido a su "juventud" y al conocimiento emergente, situación que seguramente continuará por décadas con el incremento en las observaciones de campo y los avances instrumentales, simulaciones y modelos numéricos. Tal subdivisión no fue considerada en el presente trabajo.

El término depósito de flujo de ceniza (ash flow) es un término que define un depósito de flujo piroclástico de pómez con un contenido de fragmentos tamaño ceniza mayor al 50\%. Sin embargo, este término ha sido erróneamente extrapolado para ser usado por ejemplo, en facies de grano fino de depósitos de flujos de bloques y ceniza. De esta manera, se recomienda no usarlo teniendo en cuenta que representa una facies de un depósito y que, además, los otros términos, tienen implicaciones genéticas mucho más específicas (como se menciona en cada definición). Ignimbrita es un término también usado para definir depósitos de flujo piroclástico de pómez. Sin embargo, por diferentes motivos, el término ha tomado acepciones en 
diferentes países o en diferentes usuarios en donde se ha relacionado principalmente con depósitos de flujos piroclásticos soldados, depósitos ácidos en composición o depósitos muy grandes, la mayoría de veces asociados a colapsos caldéricos. Este término ha sido ampliamente tratado aun desde principios el siglo XX como lo reporta Cook (1959). Más recientemente, Branney \& Kokelaar (2003) presentan un análisis detallado de las ignimbritas y su sedimentación asociada partiendo del hecho de que se trata de un depósito de flujo piroclástico de pómez. En nuestra propuesta se mantiene lo mencionado por estos autores independiente de las características ampliamente mencionadas y relacionadas con la ignimbrita (grado de soldamiento, vitrofídica, presencia o no de disyuncion columnar) siendo la descripción del depósito lo que imprime su condición de sedimentación y estructuras desarrolladas. Definiciones y características sobre las ignimbritas pueden ser encontradas también en Smith (1960), Spark et al. (1973), Fisher \& Schmincke (1984), Cas \& Wright (1987), Freundt et al. (2000) como se complementa en las definicies en el presente texto.

Definiciones antiguas diferenciaron los depósitos de oleadas piroclásticas asociadas a la porción inferior de un flujo piroclástico como depósitos de ground surge (literalmente oleadas de terreno), y los depósitos resultantes de la elutriación de las porciones más finas de un flujo y depositados sobre o adyacentes a flujos piroclásticos como ash cloud surge (literalmente oleadas de nube de ceniza) (Fisher, 1979; Cas \& Wright, 1987). Sin embargo, a la luz de estudios recientes (ver Branney \& Kokelaar, 2003 y referencias allí citadas), estas definiciones pueden considerarse obsoletas, por lo que se sugiere discutir los mecanismos de emplazamiento en términos de la definición de corrientes de densidad piroclástica o de facies diluidas asociadas a los flujos piroclásticos.

Los depósitos de avalanchas de escombros han sido tradicionalmente diferenciados con base en su origen en términos de si el flujo está asociado a una erupción magmática o hidromagmática (tipo Bezymianny), si está asociado a una erupción freática o hidrotermal (tipo Banday) o si no está asociado a actividad volcánica y es generada por un sismo (tipo Unzen) (Ui et al., 2000). Esta clasificación puede ser poco práctica, principalmente cuando no se está relacionado con los nombres de los volcanes que la definen. Adicionalmente, cuando el mecanismo disparador de la avalancha no es una erupción, puede no ser posible su asociación con un sismo directamente. De esta manera es que se sugiere usar los términos de sin-eruptivo o post-eruptivo para otorgar una clasificación más precisa en términos de su relación o no con una erupción de cualquier tipo.

Para autores como Beverage \& Culbertson (1964) y Pierson \& Costa (1987), los flujos de escombros tienen una concentración de sedimentos $>80 \%$ en peso y $>60 \%$ en volumen, mientras que los flujos hiperconcentrados entre $40-80 \%$ en peso y $20-60 \%$ en volumen. A partir de nuevos estudios, Smith \& Lowe (1991) propusieron valores un poco diferentes siendo 70 $90 \%$ en peso o $50-80 \%$ en volumen de concentración de sedimentos para los flujos de escombros y $55-80 \%$ en peso o $35-60 \%$ en volumen para los flujos hiperconcentrados. No obstante, análisis posteriores (ver Pierson, 2005) muestran como los límites no son tan claros debido a que no dependen exclusivamente de la concentración de los sedimentos. Por esta razón se sugiere no considerar estos límites como definitivos para la subdivisión.

Por otra parte, el término lahar con sus subdivisiones, han sido términos referidos de diversas maneras. En español se reconocen principalmente los términos de flujo de lodo, avalancha de lodo y flujo de detritos, aunque en inglés Pierson (com.. escrita, 2011) encontró muchos más (debris flood, debris slide, debris torrent, flow slide, hiperconcentrated flow, landslide, liquefaction flow, mud avalanche, mudflow, mudslide, mudspate). En este trabajo se propone unificar el uso de lahar y sus subdivisiones (flujo de escombros, flujo hiperconcentrado y flujo de corriente) con el calificativo de volcánico, con el objetivo de evitar y/o seguir ampliando las confusiones existentes hasta el momento.

En muchas ocasiones pueden confluir diferentes procesos de formación de depósitos en una erupción y, por ende, producirse una sucesión de éstos que estrictamente tendrían un 
nombre diferente; en este caso se sugiere para la descripción, la utilización del término facies de... a nivel de afloramiento para hacer referencia al depósito como se presenta en cada lugar específico. Es igualmente importante anotar que el grado de detalle descriptivo de uno o varios depósitos varía según el objetivo del estudio; no obstante, se recomienda llevar a cabo análisis texturales cuantitativos de los fragmentos y depósitos volcaniclásticos. Tales análisis ayudarían a realizar una mejor definición (ver una descripción exhaustiva en Dellino et al., 2005, Sarocchi et al., 2005 y Sarocchi et al., 2008).

De igual modo, una vez identificado un depósito, con el fin de establecer su origen, se debe de tener en cuenta una serie de parámetros que incluyan su distribución areal, forma de dispersión (variación lateral y vertical de facies), volúmenes, relaciones de altura y longitud, etc. Igualmente relevante es tener en cuenta que para cada depósito específico, pueden existir diversas posibilidades de interpretación de su origen de acuerdo a los parámetros definidos. Para depósitos muy antiguos, la principal limitación es la identificación del origen de los fragmentos (debido a procesos de meteorización, diagénesis o metamorfismo), y la verificación de la extensión y continuidad del depósito original.

Para el establecimiento de la amenaza volcánica es importante diferenciar los depósitos sineruptivos, post-eruptivos y no-eruptivos, ya que un reconocimiento inadecuado influye en la interpretación del área que podría ser afectada y de la probabilidad de ocurrencia de los diferentes fenómenos volcánicos. No obstante, existen muchas dificultades en el estudio estratigráfico de la actividad volcánica en términos del reconocimiento de la simultaneidad de los eventos caracterizados a partir de los depósitos generados.

La terminología propuesta en este trabajo para la identificación de fragmentos y depósitos volcaniclásticos ha sido usada en cursos de campo y en diversos proyectos de instituciones como Universidad de Caldas (Colombia), Servicio Geológico de Colombia, Universidad de Costa Rica, Instituto Costarricense de Electricidad y Universidad de Salta (Argentina), donde se han estudiado ampliamente sus posibilidades de aplicación, lo que ha constituido un verdadero marco de discusión para la definición y unificación de criterios durante la descripción de los depósitos volcaniclásticos. No obstante, es claro que esta discusión seguirá siendo parte del día a día en el desarrollo de la reciente ciencia de la vulcanología.

\section{AGRADECIMIENTOS}

A Thomas Pierson por las extensas discusiones acerca de la clasificación de los depósitos volcaniclásticos, y por los comentarios y sugerencias en la versión final de este manuscrito. Igualmente, agradecemos las acertadas revisiones de Katrin Sieron (Centro de Ciencias de la Tierra, Universidad Veracruzana, Xalapa, México), Gerardo Carrasco (Centro de Geociencias, Universidad Nacional Autónoma de Mexico, Juriquilla, México) e Ivan Petrinovic (CONICET - Museo de Ciencias Naturales, Universidad de Salta, Mendoza, Argentina).

\section{REFERENCIAS}

ALVARADO, G.E. \& SOTO, G.J., 2002: Pyroclastic flows generated by craterwall collapse and outpouring of the lava pool of Arenal volcano, Costa Rica.- Bull. Volcanol. 63: 557-568.

ALVARADO, G.E., SOTO, G.J., SCHMINCKE, H.-U., BOLGE, L.L. \& SUMITA, M., 2006: The 1968 andesitic lateral blast eruption at Arenal volcano, Costa Rica.- J. Volcanol. Geotherm. Res. 157: 9-33.

ARAÑA, V. \& ORTIZ, R., 1984: Volcanología.510 págs. Servicio Publicaciones Consejo superior de Investigaciones Científicas y Rueda. Madrid.

BELOUSOV, A., 1996: Deposits of the 30 March 1956 directed blast at Bezymianni volcano, Kamchatka, Rusia.- Bull. Volcanol. 57: 649-662. 
BEVERAGE, J.P. \& CULBERTSON, J.K., 1964: Hyperconcentrations of suspended sediment.- Amer. Soc. Civil Engineers Proc., Hydraulics Division J. 90: 117-128.

BORRERO, C., ROSERO, J.S., VALENCIA, J.D. \& PARDO, A., 2008: La secuencia volcaniclástica de Aranzazu: Registro del impacto del volcanismo en un sistema fluvial Neógeno en la parte media de la Cordillera Central, Colombia.- Bol. Geología, Univ. Industrial de Santander, 30: 61-77.

BOUVET DE MAISONNEUVE, C., BACHMANN, O. \& BURGISSER, A., 2009: Characterization of juvenile pyroclasts from the Kos Plateau Tuff (Aegean Arc): insights into the eruptive dynamics of a large rhyolitic eruption.Bull. Volcanol. 71: 643-658.

BRANNEY, M.J. \& KOKELAAR. P., 2003: Pyroclastic density currents and the sedimentation of ignimbrites.- Geol. Soc. London, Memoirs, 27: 1-143.

BRANNEY, M.J., BONNICHSEN, B., ANDREWS, G.D.M., ELLIS, B., BARRY, T.L. \& MCCURRY, M., 2008: "Snake River (SR)-type" volcanism at the Yellowstone hotspot track: distinctive products from unusual, high-temperature silicic supereruptions.- Bull.Volcanol. 70: 293-314.

BROWN, R.J., BONADONNA, C. \& DURANT, A.J., 2011: A review of volcanic ash aggregation.- Phys. Chemist. Earth, DOI:10.1016/j.pce.2011.11.001

BÜTTNER, R., DELLINO, P., LA VOLPE, L., LORENZ, V., \& ZIMANOWSKI, B., 2002: Thermohydraulic explosions in phreatomagmatic eruptions as evidenced by the comparison between pyroclasts and products from Molten Fuel Coolant Interaction experiments.- J. Geophys. Res. 107, 2277, doi: 10.1029/2001JB000511.
CAS, R.A.F. \& WRIGHT, J.V., 1987: Volcanic successions, modern and ancient.- 546 págs., Allen and Unwin Ltd., London.

CAS, R.A.F., HAYMAN, P., PITTARI, A. \& PORRITT, L., 2008: Some major problems with existing models and terminology associated with kimberlite pipes from a volcanology perspective, and some suggestions.- J. Volcanol. Geotherm. Res. 174: 209-225.

CASHMAN, K.V. \& MANGAN, M.T., 1994: Physical aspects of magmatic degassing II. Constraints on vesiculation processes from textural studies of eruptive products.- En: CARRILL, M.R., \& HOLLOWAY, J.R. (eds): Volatiles in magmas.- Rev. Mineral. 30: 447-478.

COOK, E.F., 1959: Ignimbrite bibliography.- 30 págs., Idaho Bur. Mines Geol., Inf. Circ., Moscow.

CROWE, B.M. \& FISHER, R.V., 1973: Sedimentary structures in base surge deposits with special reference to cross bedding; Ubehebe Crater, Death Valley, California.- Geol. Soc. Amer. Bull. 84: 663-682.

DASGUPTA, P., 2003: Sediment gravity flow-the conceptual problems.- Earth-Science Rev. 62: $265-281$

DELliNO, P., MELE, D., BONASIA, R., BRAIA, G., LA VOLPE, L. \& SULPIZIO, R., 2005: The analysis of the influence of pumice shape on its terminal velocity.- Geophys. Res. Lett., 32, L21306, doi:10.1029/2005GL023954.

DORONZO, D.M., 2012: Two new end members of pyroclastic density currents: Forced convection-dominated and inertiadominated.- J. Volcanol. Geotherm. Res. 219-220: 87-91. 
DRUITT, T.H., 1998: Pyroclastic density currents.- En: GILBERT, J.S. \& SPARKS, R.S.J. (eds.): The physics of explosive volcanic eruptions.- Geol. Soc. London, Sp. Publ. 145: 145-182.

FISHER, R.V., 1961: Proposed classification of volcaniclastic sediments and rocks.- Geol. Soc. Amer. Bull. 72: 1409-1414.

FISHER, R.V., 1966: Mechanism of deposition from pyroclastic flows.- Amer. J. Sci. 264: 350-363.

FISHER, R.V. \& WATERS, A., 1970: Base surge bed forms in maar volcanoes.- Amer. J. Sci. 268: 157-180.

FISHER, R.V., 1979: Models for pyroclastic surges and pyroclastic flows.- J. Volcanol. Geotherm. Res. 6: 305-318.

FISHER, R.V. \& SCHMINCKE, H.-U., 1984: Pyroclastic rocks.- 472 págs., SpringerVerlag, Berlín.

FISHER, R.V., GLICKEN, H.X. \& HOBLITT, R.P., 1987: May 18, 1980. Mount St. Helens deposits in South Coldwater Creek, Washinghton.- J. Geophys. Res. 92(B10): 10267-10283

FISHER, R.V. \& SMITH, G.A., 1991: Volcanism, tectonics and sedimentation.- En: FISHER, R.V. \& SMITH, G.A. (eds.): Sedimentation in Volcanic Settings.- Soc. Sediment. Geol., Spec. Publ.: 1-5.

FOLK, R.L. \& WARD W.C., 1957: Brazos river bar: a study of the significance of grain size parameters.- J. Sediment. Petrol. 27: 3-26.

FREUNDT, A. \& BURSIK, M.I., 1998: Pyroclastic flow transport mechanisms.En: FREUNDT, A. \& ROSI, M., (eds.): From magma to tephra, modeling physical processes of explosive volcanic eruptions.Elsevier, Amsterdam: 173 - 231.

FREUNDT, A. \& SCHMINCKE, H.-U., 1985: Lithic-enriched segregation bodies in pyroclastic flow deposits of Laacher See volcano (East Eifel, Germany).- J. Volcanol. Geotherm. Res. 25: 193-224.

FREUNDT, A., WILSON, C.F.N. \& CAREY, S.N., 2000: Ignimbrites and block and ash flow deposits.- En: SIGURDSSON, H. (ed.): Encyclopedia of Volcanoes.Academic Press: 581-600.

GARDNER, J.E, THOMAS, R.M.E., JAUPART, C. \& TAIT, S., 1996: Fragmentation of magma during Plinian volcanic eruptions.Bull. Volcanol. 58: 144-162.

GIACHETTI, T., BURGISSER, A., ARBARET, L., DRUITT, T.H. \& KELFOUN, K., 2011: Quantitative textural analysis of Vulcanian pyroclasts (Montserrat) using multi-scale X-ray computed microtomography: comparison with results from 2D image analysis.- Bull. Volcanol. 73: 1295-1309.

GILBERT, J.S. \& SPARKS, R.S.J., 1998: Future research directions on the physics of explosive volcanic eruptions.- En: GILBERT, J.S., \& SPARKS, R.S.J. (eds.): The Physics of Explosive Volcanic Eruptions.- Geol. Soc. Spec. Publ. 145: 1-8.

GILLESPIE, M.R. \& STYLES, M.T., 1999. BGS Rock Classification Scheme. Volume 1. Classification of igneous rocks [2 $2^{\mathrm{da}}$ ed.].British Geol. Survey Res. Rep. RR 99-06. U.K.

HEIKEN， G. \& WOHLETZ, K.H., 1985: Volcanic ash.- 246 págs. Berkeley, Univ. California Press.

HOUGHTON, B.F. \& WILSON, C.J.N., 1989: A vesicularity index for pyroclastic deposits.- Bull. Volcanol. 51: 451-462. 
INGRAM, R.L., 1954: Terminology for the thickness of stratification and parting units in sedimentary rocks.- Geol. Soc. Amer. Bull. 65: 937-938.

IVERSON, R.M., 1997: The physics of debris flow.- Reviews Geophys. 35: 245-296.

IVERSON, R.M. \& VALLANCE, J.W., 2001: News views of granular mass flows.Geology, 29: 115-118.

JERRAM, D.A. \& STOLLHOFEN, H., 2002: Lava-sediment interaction in desert settings; are all peperite-like textures the result of magma-water interaction?- J. Volcanology Geotherm. Res. 114: 231249.

KIEFFER, S., 1981: Fluid dynamics of the May 18 blast at Mt. St. Helens.- En: LIPMAN, P.W. \& MULLINEAUX, R.R. (eds.): The 1980 eruption of Mount St. Helens, Washington.- US Geol. Surv., Profess. Paper, 1250: 379-400.

KLUG, C., \& CASHMAN, K.V., 1994: Vesiculation of May 18, Mount St. Helens pumice.- Geology, 22: 468-472.

KLUG, C. \& CASHMAN, K.V., 1996: Permeability development in vesiculating magmas: implications for fragmentation.Bull. Volcanol. 58: 87-100.

MADER, H.M., 1998: Conduit flow and fragmentation.- En: GILBERT, J.S. \& SPARKS, R.S.J. (eds.): The Physics of Explosive Volcanic Eruptions.- Geol. Soc. Spec. Publ. 145: 51-72.

MANGAN, M.T. \& CASHMAN, K.V., 1996: The structure of basaltic scoria and reticulite and inferences for vesiculation, foam formation, and fragmentation in lava fountains.- J. Volcanol. Geotherm. Res. 73: 1-18.
MANVILLE, V., NÉMETH, K. \& KANO, K., 2009: Source to sink: A review of three decades of progress in the understanding of volcaniclastic processes, deposits, and hazards.- Sediment. Geology, 220: 136-161.

MARTÍNEZ, R.M., LAGO, M., VAQUER, R., ARRANZ, E. \& VALENZUELA, J.I., 1996: Precisiones vulcanológicas entre mecanismos de fragmentación y emplazamiento de rocas volcanoclásticas.- Gaceta, 20: $515-517$.

MAZZONI, M.M., 1985: Procesos y depósitos piroclásticos.- Asociac. Geol. Argentina, Serie "B", 14: 1-115.

MCPHIE, J., DOYLE, M.G. \& ALLEN, R.L., 1993: Volcanic Textures.- 196 págs. CODES, Univer. Tasmania, Hobart.

MIYABUCHI, Y., WATANABE K., \& EGAWA, Y., 2006: Bomb-rich basaltic pyroclastic flow deposit from Nakadake, Aso Volcano, southwestern Japan.- J. Volcanol. Geotherm. Res. 155: 90-103.

MOORE, 1967: Base surges in recent volcanic eruptions.- Bull. Volcanol. 30: 337-363.

NAIRN, I.A, WOOD, C.P. \& HEWSON, C.A.Y., 1979: Phreatic eruptions of Ruapehu: April 1975.- New Zealand J. Geol. Geophys. 22: 155-170.

NAVON, O. \& LYAKHOVSKY, V., 1998: Vesiculation processes in silicic magmas.En: GILGER, J.S. \& SPARKS, R.S.J. (eds.): The Physics of Explosive Volcanic Eruptions. Geol. Soc. Spec. Public. 145: 27-50.

NÉMETH, K. \& WHITE, C.M., 2009: Intravent peperites related to the phreatomagmatic 71 Gulch volcano, western Snake River Plain volcanic field, Idaho (USA).- J. Volcanol. Geotherm. Res. 183: 30-41. 
PETRY, K., JERRAM, D.A., ALMEIDA, D.P.M. \& ZERFASS, H., 2007: Volcanicsedimentary features in the Serra Geral Fm., Paraná Basin, southern Brazil: examples of dynamic lava-sediment interactions in an arid setting.- J. Volcanol. Geotherm. Res. 159: 313-325.

PETTIJOHN, E.J., 1973: Sedimentary rocks [2 $2^{\text {da }}$ ed.].- 628 págs., Harper and Row, Publishers, Nueva York.

PIERSON, T.C. \& COSTA, J.E., 1987: Arheologic classification of subaerial sediment - water flows.- Geol. Soc. Amer. Rev. Engine. Geol. 7: 1-12

PIERSON, T., 2005: Hyperconcentrated flow transitional process between water flow and debris flow.- En: JAKOB, M. \& HUNGR, O. (eds.): Debris-flow hazards and related phenomena.- Springer Parxis Books: 159-202.

POLACCI, M., BAKER, D.R., MANCINI, L., TROMBA, G. \& ZANINI, F., 2006: Threedimensional investigation of volcanic textures by X-ray microtomography and implications for conduit processes.Geophys. Res. Lett., 33, L13312, DOI:10.1029/2006GL026241

RODOLFO, K.S. \& ARGUDEN, A.T., 1991: Rain-lahar generation and sedimentdelivery systems at Mayon volcano, Philippines.- En: FISCHER, R.V. \& SMITH, G.A. (eds): Sedimentation in Volcanic Settings.- Soc. Sediment. Geol., Spec. Publ., 45: 71-87.

RUST, A.C. \& CASHMAN, K.V., 2004: Permeability of vesicular silicic magma: inertial and hysteresis effects.- Earth Planetary Sci. Lett. 228: 93-107.

RUSH, A., CASHMAN, K.V. \& WRIGHT, H.M., 2009: Bubble expansion, gas percolation and preservation of pyroclasts.- [resumen] Amer. Geophys. Union, Fall meeting, V11F-01.

SAROCCHI, D., BORSELLI, L. \& MACÍAS, J.L., 2005: Construcción de perfiles granulométricos de depósitos piroclásticos por métodos ópticos.- Rev. Mexicana de Ciencias Geol. 22: 371-382.

SAROCCHI, D., BORSELLI, L. \& MACÍAS, J.L., 2008: New tools to investigate textures of pyroclastic deposits.Collapse Calderas Workshop, IOP Conference Series.- Earth Environm. Sci. 3, 012009, DOI:10.1088/17551307/3/1/012009.

SCHIPPER, C.I., WHITE, J.D.L., HOUGHTON, B.F., SHIMIZU, N., STEWART, R.B., 2010: "Poseidic" explosive eruptions at Loihi Seamount, Hawaii.- Geology, 38: 291-294.

SCHMID, R., 1981: Descriptive nomenclature and classification of pyroclastic deposits and fragments: Recommendations of the IUSG Subcommission on the Systematics of Igneous Rocks.- Geology, 9: 41-43.

SCHMINCKE, H.-U. \& VAN DEN BOGAARD, P., 1991: Tephra Layers and Tephra Events.- En: EINSELE, G., RICHKEN, W. \& SEILACHER, A. (eds.): Cycles and events in stratigraphy.- Springer-Verlag, Berlín: 392-429.

SCOLAMACCHIA, T. \& MACÍAS, J.L., 2005: Distribution and stratigraphy of deposits produced by diluted pyroclastic density currents of the 1982 eruption of El Chichón volcano, Chiapas, México.- Rev. Mexicana Ciencias Geol. 22: 159-180.

SCOLAMACCHIA, T., MACÍAS, J.L., SHERIDAN, M.F. \& HUGHES, S.R., 2005: Morphology of ash aggregates from wet pyroclastic surges of the 1982 eruption of El Chichón Volcano, Mexico.- Bull. Volcanol. 68: 171-200. 
SHEA, T., HOUGHTON, B.F., GURIOLI, L., CASHMAN, K.V., HAMMER, J.E. \& HOBDEN, B.J., 2010: Textural studies of vesicles in volcanic rocks: An integrated methodology.- J. Volcanol. Geotherm. Res. 190: 271-289.

SHERIDAN, M.F. \& WOHLETZ, K.H., 1983: Hydrovolcanism: basic considerations and review.- J. Volcanol. Geotherm. Res. 17: 1-29.

SIEBERT, L., 1984: Large volcanic debris avalanches: Characteristics of source areas, deposits, and associated eruptions.- J. Volcanol. Geotherm. Res. 22: 163-197.

SKILLING, I.P., WHITE, J.D.L. \& MCPHIE, J., 2002. Peperite: a review of magmasediment mingling.- J. Volcanol. Geotherm. Res. 114: 1-17.

SMITH, R.L., 1960: Zones and zonal variations in welded ash flows.- U. S. Geol. Surv. Profess. Paper 354-F: 149-159.

SMITH, G.A. \& FRITZ, W.J., 1989: Volcanic influences on terrestrial sedimentation.Geology, 17: 375-376.

SMITH, G.A. \& LOWE, D.R., 1991: Lahars: volcano - hydrologic events and deposition in the debris flow-hyperconcentrated flow continuum.- En: FISHER, R.V. \& SMITH, G.A. (eds.): Sedimentation in Volcanic Settings.- Soc. Sediment. Geol., Spec. Publicat. 45: 60-70.

SOHN, Y.K., \& COUGH, S.K., 1989: Depositional processes of the Suwolbong tuff ring, Cheju Island (Korea).- Sedimentol. 36: 837-855.

SOHN, Y.K., PARK, K.H., \& YOON, S.H., 2008: Primary versus secondary and subaereal versus submarine hydrovolcanic deposits in the surface of Jeju Island, Korea.- Sedimentol. 55: 899-924.
SPARK, R.S.J., SELF, S. \& WALKER, G.P.L., 1973: Products of ignimbrite eruptions.Geology, 1: 115-118.

THOMAS, N., JAUPART, C. \& VERGNIOLLE, S. 1994: On the vesicularity of pumice.- J. Geophys. Res. 99: 15633-15644

TILLING, R., 1993: Los peligros volcánicos.125 págs., Organización Mundial de observatorios vulcanológicos (WOVO), Santa Fé.

UDDEN, J.A., 1914. Mechanical composition of clastic sediments.- Geol. Soc. Amer. Bull. 25: 655-744.

UI, T., TAKARADA, S. \& YOSHIMOTO, M., 2000: Debris avalanches.- En: SIGURDSSON, H. (ed.): Encyclopedia of Volcanoes.- Academic Press: 617-626.

VALENTINE, G.A. \& FISHER, R.V., 2000: Pyroclastic surges and blasts.- En: SIGURDSSON, H. (ed.), Encyclopedia of Volcanoes.- Academic Press: 571-580.

VALLANCE, J.W., 2000: Lahars.- En: SIGURDSSON, H. (ed.): Encyclopedia of Volcanoes.- Academic Press: 601-625.

VALLANCE, J.M. \& SCOTT, K.M., 1997: The Osceola mudflow from Mount Rainier: Sedimentology and hazard implications of a huge clay - rich debris flow.- Geol. Soc. Amer. Bull. 9: 143-163.

VAN EATON, A.R., MUIRHEAD, J.D., WILSON, C.J.N. \& CIAMARELLI, C., 2012: Growth of volcanic ash aggregates in the presence of liquid water and ice: an experimental approach.- Bull. Volcanol. 74: 1963-1984.

VINCENT, P., 2000: Volcanoes: from magmas to tephras and epivolcaniclastics.En: LEYRIT, H., \& MONTENANT, C. (eds.): Volcaniclastic rocks, from mag- 
mas to sediments.- Gordon and Breach Science Publishers: 1-32.

WADELL, H.A., 1933: Sphericity and roundness of rock particles.- J. Geology, 41: 310-331.

WAICHEL, B.L., DE LIMA, E.F., SOMMER, C.A. \& LUBACHESKY, R., 2007: Peperite formed by lava flows over sediments: An example from the central Paraná Continental Flood Basalts, Brazil.- J. Volcanol. Geotherm. Res. 159: 343-354.

WALKER, G.P.L., 1971: Grain-size characteristics of pyroclastic deposits.- J. Geology, 30: 377-392.

WATTON, T.J., JERRAM, D.A., THORDARSON, T. \& DAVIES, R.J., 2013: Three-dimensional lithofacies in hyaloclastite deposits.- J. Volcanol. Geotherm. Res. 250: 19-33.

WENTWORTH, C.K., 1922: A scale of grade and class terms for clastic sediment. J. Geology, 30: 377-392.

WENTWORTH, C.K. \& WILLIAMS, H., 1932: The classification and terminology of the pyroclastic rocks.- Bull. Nat. Res. Council, Report of the Commis. Sedimentation, 89: 19-53.

WHITE, J.D.L., MCPHIE, J. \& SKILLING, I., 2000: Peperite: A useful genetic term.Bull. Volcanol. 62: 65-66.

WHITE, J.D.L. \& HOUGHTON, B.F., 2006: Primary volcaniclastic rocks.- Geology, 34: 677-680.

WHITHMAN A.G. \& SPARKS, R.S.J., 1986: Pumice.- Bull. Volcanol. 48: 209-223.

WILSON, C.J.N. \& HOUGHTON, B.F., 2000: Pyroclastic transport and deposition.- En: SIGURDSSON, H. (ed.): Encyclopedia of Volcanoes.- Academic Press: 545-554.

WOHLETZ, K.H., 1983: Mechanisms of hydrovolcanic pyroclastic formation: grain-size, scanning electron microscopy, and experimental studies.- J. Volcanol. and Geotherm. Res. 17: 31-63.

WOHLETZ, K.H., 1998: Pyroclastic surges and compressible two-phase flow.- En: FREUND, A. (ed.). From magma to tephra: Modelling physical processes of explosive volcanic eruptions.- Elsevier Science Publications, Amsterdam: 247-312. 
